\title{
LINHA BÁSICA DE SUSCETIBILIDADE DA LAGARTA MILITAR A INSETICIDAS POR INGESTÃO NA CULTURA DO MILHO
}

\author{
Gustavo Storch* \\ Alci Enimar Loeck** \\ Deivid Araújo Magano*** \\ Mateus Batista Remor ${ }^{* * * *}$
}

RESUMO: Dentre os cereais cultivados no Brasil, o milho é o mais expressivo, já obtendo áreas com produtividades superiores a 16 toneladas por hectare. No entanto, essa expressão do seu potencial tem sido reduzida em consequência de severos ataques de pragas, dentre as quais se destaca a lagarta militar, que promove sérios danos à cultura. $\mathrm{O}$ objetivo desse trabalho foi estudar a linha básica de suscetibilidade aos inseticidas (lufenurom, novalurom, methoxifenizida e espinosade) sobre a população de lagartas de Spodoptera frugiperda. Foram realizados bioensaios para a obtenção das curvas de concentração-resposta para lagartas de terceiro instar, onde cubos de dieta $(1,5 \times 2,5 \mathrm{~cm})$, artificial descrita por Greene et al. (1976), foram submersos na calda com cada inseticida durante um segundo e imediatamente colocados em tubos de vidro de $2,5 \times 8,5 \mathrm{~cm}$ previamente esterilizados. Logo após as lagartas foram individualizadas nos mesmos tubos, os quais foram tamponados com algodão hidrófugo, e mantidos em câmara climatizada tipo BOD à temperatura de $25^{\circ} \mathrm{C}$ e fotofase de 14 horas, até a pupação. Os dados de mortalidade foram registrados diariamente até o estágio pupal. As curvas foram determinadas através da análise de Probit. Desse modo, foi possível observar que o inseticida lufenurom apresentou CL50 $=0,265 \mathrm{ppm}$, novalurom CL50 $=0,066$ ppm, metoxifenozida CL50 $=4,544 \mathrm{ppm}$ e espinosade CL50 $=0,970 \mathrm{ppm}$.

PALAVRAS-CHAVE: Controle químico; Pesticidas; Zea mays.

\footnotetext{
Doutor em Fitossanidade pela Universidade Federal de Pelotas (UFPel); Pesquisador do Desenvolvimento de produtos Syngenta, Curitiba (PR), Brasil.

** Doutor em Entomologia pela Universidade de São Paulo (USP/SP); Docente Titular no Departamento de Fitossanidade (FAEM-UFPel), Pelotas (RS), Brasil.

*** Doutorando do Programa de Pós graduação em Engenharia Agrícola, na área de Engenharia ambiental de Agroecossistemas Universidade Federal de Santa Maria (UFSM), Santa Maria (RS), Brasil;

E-mail: maganodeivid@gmail.com

**** Engenheiro agrônomo, Departamento de fitossanidade FAEM/UFPEL Pelotas (RS), Brasil.
} 


\title{
SUSCEPTIBILITY OF ARMY WORM TO INSECTICIDES BY INGESTION IN CORN CROPS
}

\begin{abstract}
Corn is one of the most relevant cereals cultivated in Brazil, with areas featuring productivity of 16 tons per hectare. However, productivity has been reduced due to several attacks by pests, particularly the fall armyworm, which seriously damage crops. Current study analyzes the basic susceptibility of insecticides (lufenurom, novalurom, methoxphenozide and spinosad) on populations of Spodoptera frugiperda. Bioassays have been undertaken for concentration-response curves for armyworms at the third instar where artificial diet cubes $(1.5 \times 2.5 \mathrm{~cm})$, described by Greene et al. (1976), were soaked in broth with each insecticide for a second and immediately placed in previously sterilized $2.5 \times 8.5 \mathrm{~cm}$ test tubes. The armyworms were individualized in the tubes which were buffered with hydrophobe cotton and maintained in a BOD chamber at $25^{\circ} \mathrm{C}$ and a 14 -hour photophase till cocoon formation. Mortality rates were reported daily till the cocoon stage and curves were calculated by Probit analysis. The insecticide lufenuron showed CL50 $=0.265 \mathrm{ppm}$; novaluron CL50 $=0.066 \mathrm{ppm}$, methoxphenozide CL50 $=4.544$ ppm and spinosad CL50 $=0.970 \mathrm{ppm}$.
\end{abstract}

KEY WORDS: Chemical control; Pesticides; Zea mays.

\section{INTRODUÇÃO}

O Brasil cultiva cerca de 15,7 milhões de hectares de milho, o que o torna o $3^{\circ}$ maior produtor mundial, atrás apenas dos Estados Unidos e da China. Os Estados do Mato Grosso e Paraná são os maiores produtores brasileiros da cultura, com 43\% da produção nacional (CONAB, 2014).

No entanto, um dos fatores responsáveis pela redução dos índices de produtividade diz respeito ao ataque de pragas. Dentre as pragas que ocorrem na cultura do milho e sorgo, a lagarta militar Spodoptera frugiperda (também denominada de lagarta do cartucho) se destaca, atacando inúmeras plantas pertencentes a diferentes famílias (SILVA et al., 1968).

A cultura do milho vem sendo prejudicada pela lagarta do cartucho há muitos anos, causando prejuízos que variam de 34,1 (CARVALHO, 1970) a 60\% 
dependendo da cultivar e estado fenológico da cultura em que ocorre o ataque (CRUZ et al., 2008).

O controle de $S$. frugiperda em milho é realizado quase que exclusivamente com inseticidas químicos (CARVALHO, 1982; GASSEN, 1994), o que ao longo do tempo promoveu resistência aos principais grupos de inseticidas (OMOTO, 2000).

Em algumas regiões brasileiras são realizadas até dez aplicações de inseticidas para o controle da lagarta do cartucho, devido à resistência desse inseto a alguns grupos de inseticidas utilizados (CRUZ et al., 1999). A importância da S. frugiperda se deve tanto pelos danos provocados como também à dificuldade de seu controle, e vem sendo alvo de pesquisas a campo com populações naturais e em estudos em laboratório, fazendo o uso de criações artificiais para estudos toxicológicos.

O método de tratamento superficial da dieta, dentre os métodos empregados em laboratório, apresenta uma série de vantagens, por sua praticidade, utilizando pequenas quantidades de produtos, produzindo resultados consistentes, e gerar a possibilidade de ser utilizado tanto na avaliação de novos produtos como também no monitoramento de populações de campo para o manejo da resistência da espéciealvo (WAQUIL et al., 2004).

A técnica de aplicação superficial de inseticidas sobre dieta artificial mostrou-se eficiente para avaliar a suscetibilidade de $S$. frugiperda aos inibidores da biossíntese de quitina e a B. thuringiensis (SCHMIDT, 2002; BARRERO, 2005). A partir desses trabalhos é possível obter informações sobre a linha básica de suscetibilidade de $S$. frugiperda, que representam o primeiro passo de um programa preventivo de manejo da resistência (SCHMIDT, 2002), bem como são informações básicas para futuros estudos sobre os efeitos deletérios de inseticidas sobre as pragas.

Nesse sentido o trabalho objetivou identificar a linha básica de suscetibilidade de S. frugiperda aos inseticidas com modo de ação por ingestão. 


\section{MATERIAIS E MÉTODOS}

Lagartas oriundas do município de Pelotas (RS) foram capturadas em área de milho e encaminhadas ao laboratório, onde foram mantidas por cinco gerações dando início a uma criação estoque. A criação estoque deu suporte com fornecimento de lagartas de terceiro instar para a realização dos testes toxicológicos.

Determinou-se para lufenurom (Match EC), novalurom (Gallaxy 100 EC), metoxifenozida (Intrepid 240SC), espinosade (Tracer), os intervalos de concentrações que ocasionam mortalidade, no estágio L3, próxima de zero até próxima de $100 \%$. Estas concentrações foram obtidas através de diluições sequenciais.

A partir das concentrações definidas previamente, foi avaliado para o inseticida lufenurom que foram utilizadas 09 concentrações dentro do intervalo entre $0,050 \mathrm{a}$ 0,625 ppm $(0,050,0,121,0,193,0,265,0,337,0,409,0,481,0,553,0,625$ ppm), para novalurom foram utilizadas 10 concentrações dentro do intervalo de entre $0,010 \mathrm{a}$ 0,150 ppm (0,010, 0,0253, 0,0411, 0,0567, 0,0722, 0,0878, 0,1033, 0,1189, 0,1344, $0,1500 \mathrm{ppm})$, para metoxifenozida foram utilizadas 05 concentrações dentro do intervalo de 2,4 a 7,2 ppm (2,40,3,60, 4,80, 6,00, 7,20 ppm), e para espinosade foram utilizadas 05 concentrações dentro do intervalo de 0,12 a 1,76 ppm $(0,120,0,530$, $0,940,1,350,1,760 \mathrm{ppm})$. A testemunha foi tratada somente com água destilada. $\mathrm{O}$ número de concentrações utilizadas para determinar a curva foi variável, conforme a disponibilidade de lagartas para o ensaio.

Os inseticidas foram diluídos em água, colocados em recipientes de $150 \mathrm{~mL}$, onde cubos de dieta $(1,5 \times 2,5 \mathrm{~cm})$ artificial descrita por Greene et al. (1976) foram submersos durante um segundo, e imediatamente colocados em tubos de vidro de $2,5 \times 8,5 \mathrm{~cm}$ previamente esterilizados durante 02 horas em estufa a $120^{\circ} \mathrm{C}$. Logo após as lagartas foram individualizadas, os quais foram tamponados com algodão hidrófobo, e mantidos em câmara climatizada tipo BOD à temperatura de $25{ }^{\circ} \mathrm{C}$ e fotofase de 14 horas, até a pupação. Os dados de mortalidade foram registrados diariamente até o estágio pupal. Os dados obtidos foram analisados pelo software Micro Probit (1986), que gerou a curva de concentração letal. 
Tabela 1. Mortalidade esperada e concentração letal mediana em ppm, dos inseticidas lufenurom, novalurom, metoxifenozida e espinosade, obtidas através da técnica de imersão de dieta artificial, fornecidas a lagartas de $3^{\circ}$ instar de Spodoptera frugiperda, utilizando para análise o software Micro Probit (1986), IC 95\%. Temperatura de $25 \pm 2{ }^{\circ} \mathrm{C}$, e fotofase de 14 horas

\begin{tabular}{ccccc}
\hline & Lufenurom & Novalurom & Metoxifenozida & Espinosade \\
\hline $\begin{array}{c}\text { Mortalidade } \\
\text { Esperada (\%) }\end{array}$ & $\begin{array}{c}\text { Concentração } \\
\text { Letal (PPM) }\end{array}$ & $\begin{array}{c}\text { Concentração } \\
\text { Letal (PPM) }\end{array}$ & $\begin{array}{c}\text { Concentração } \\
\text { Letal (PPM) }\end{array}$ & $\begin{array}{c}\text { Concentração } \\
\text { Letal (PPM) }\end{array}$ \\
\hline 5 & 0,101 & 0,010 & 1,635 & 0,142 \\
10 & 0,125 & 0,015 & 2,049 & 0,217 \\
15 & 0,144 & 0,020 & 2,386 & 0,289 \\
20 & 0,162 & 0,025 & 2,694 & 0,363 \\
25 & 0,179 & 0,030 & 2,989 & 0,441 \\
30 & 0,195 & 0,036 & 3,281 & 0,526 \\
35 & 0,212 & 0,042 & 3,577 & 0,619 \\
40 & 0,229 & 0,049 & 3,883 & 0,722 \\
45 & 0,246 & 0,057 & 4,203 & 0,838 \\
50 & $\mathbf{0 , 2 6 5}$ & $\mathbf{0 , 0 6 6}$ & $\mathbf{4 , 5 4 4}$ & $\mathbf{0 , 9 7 0}$ \\
55 & 0,286 & 0,076 & 4,912 & 1,124 \\
60 & 0,308 & 0,088 & 5,317 & 1,304 \\
65 & 0,333 & 0,103 & 5,771 & 1,522 \\
70 & 0,361 & 0,121 & 6,292 & 1,790 \\
75 & 0,394 & 0,144 & 6,908 & 2,134 \\
80 & 0,435 & 0,174 & 7,664 & 2,594 \\
85 & 0,488 & 0,218 & 8,651 & 3,258 \\
90 & 0,563 & 0,288 & 10,075 & 4,339 \\
95 & $\mathbf{0 , 6 9 7}$ & $\mathbf{0 , 4 3 7}$ & $\mathbf{1 2 , 6 2 8}$ & $\mathbf{6 , 6 3 5}$ \\
99 & 1,040 & 0,953 & 19,280 & 14,702 \\
\hline
\end{tabular}

Fonte: Dados da pesquisa

O inseticida lufenurom apresentou CL50 $=0,265$ ppm (I. C. 95\% 0,215 0,309 ) e CL95 = 0,697 ppm (I. C. 95\% 0,553 - 1,071); novalurom CL50 = 0,066 ppm (I. C. 95\% 0,049-0,084) e CL95 = 0,437 ppm (I. C. 95\% 0,256 - 1,447), metoxifenozida apresentou CL50 = 4,544 ppm (I. C. 95\% 3,569 - 5,574) e CL95 = 12,628 ppm (I. C. $95 \%$ 8,681 - 41,184), espinosade CL50 = 0,970 ppm (I. C. 95\% 0,638 - 1,382) e CL95 = 6,63 ppm (I. C. 95\% 3,195 - 96,545) (Tabelas 1 e 2). Desta forma, é possivel 
determinar as doses de acordo com o índice de mortalidade desejado para estudar o efeito de sub-dose dos respectivos inseticidas, estão representada graficamente as curvas de concetração-resposta nas Figuras 1, 2, 3 e 4.

Em estudo sobre a linha básica de suscetibilidade de $S$. frugiperda ao inseticida lufenurom, Schmidt (2002) obteve valores superiores para CL50 e CL95 0,765 e 2,856 ppm, respectivamente. Essa diferença deve-se, provavelmente, ao curto período de avaliação que foi no máximo a 120 horas após a aplicação e também a variação na forma de aplicação do inseticida sobre a superfície.

O inseticida lufenurom, inibidor da síntese de quitina, promove mortalidade durante toda a fase de desenvolvimento larval, não só poucas horas após a aplicação, e esse também pode ser um dos motivos dessa variação. Além disso, diferenças metodológicas também podem ter contribuído para as diferenças entre os valores observados nos respectivos estudos. No estudo conduzido por Schmidt (2002) o autor depositou o inseticida somente na superfície da dieta, enquanto que no presente estudo, todas as superfícies externas da dieta foram expostas ao princípio ativo.

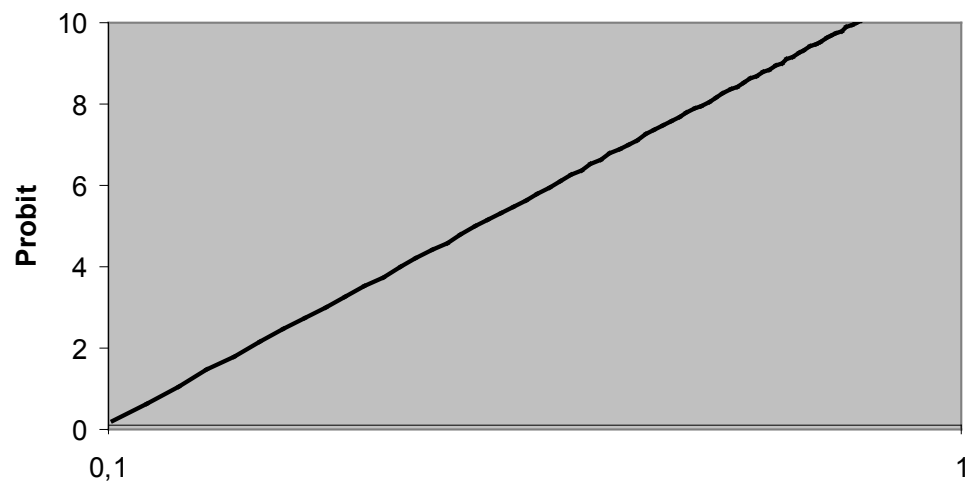

PPM de lufenurom em água destilada

Figura 1. Curva de concentração-resposta de lagartas de $3^{\circ}$ instar de $S$. frugiperda a lufenurom até a fase de pupa em bioensaio usando a técnica de imersão da dieta 


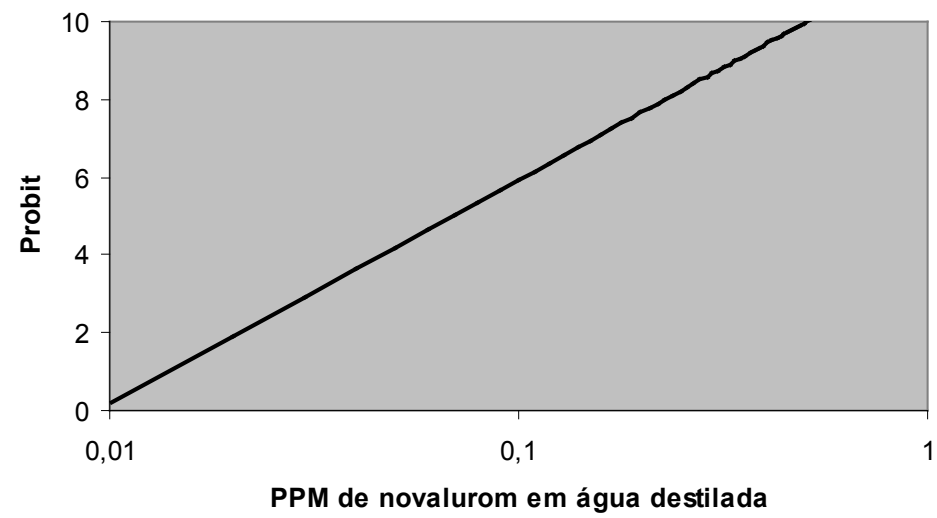

Figura 2. Curva de concentração-resposta de lagartas de $3^{\circ}$ instar de $S$. frugiperda a novalurom até a fase de pupa em bioensaio usando a técnica de imersão da dieta

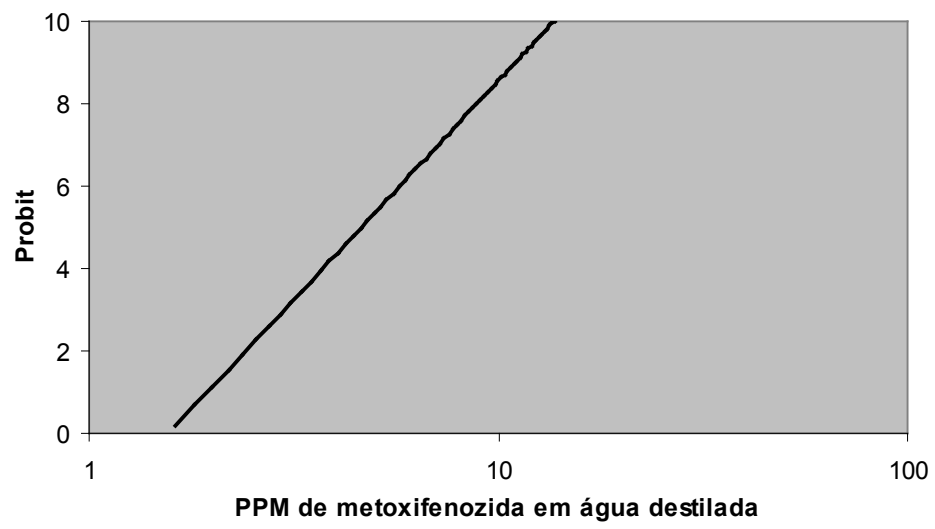

Figura 3. Curva de concentração-resposta de lagartas de $3^{\circ}$ instar de $S$. frugiperda a metoxifenozida até a fase de pupa em bioensaio usando a técnica de imersão da dieta 


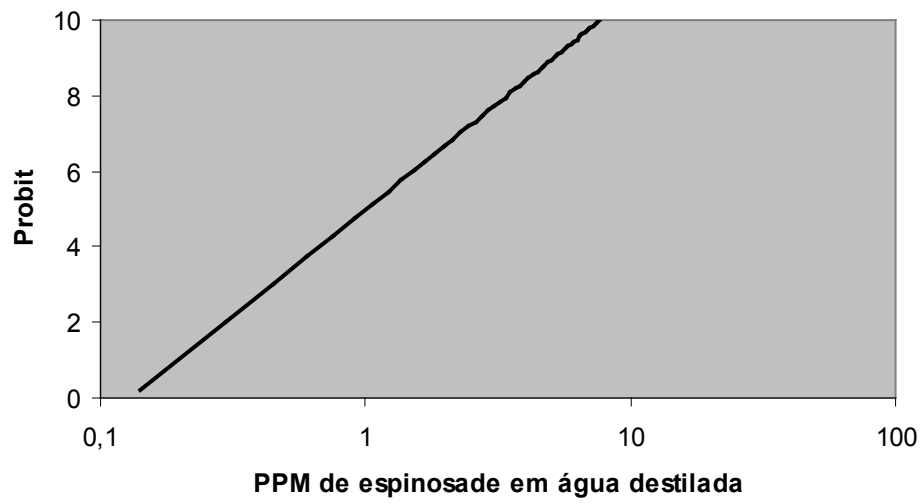

Figura 4. Curva de concentração-resposta de lagartas de $3^{\circ}$ instar de $S$. frugiperda a espinosade até a fase de pupa em bioensaio usando a técnica de imersão da dieta

Tabela 2. Resposta de lagartas de $3^{\circ}$ instar de $S$. frugiperda a lufenurom, novalurom, metoxifenozida e espinosade em bioensaio usando a técnica de aplicação por imersão de dieta artificial. Temperatura de $25 \pm 2{ }^{\circ} \mathrm{C}$, e fotofase de 14 horas. UFPel/FAEM, 2005

\begin{tabular}{lcccccc}
\hline \multicolumn{1}{c}{ Inseticida } & $\mathrm{n}^{\mathrm{a}}$ & $\begin{array}{c}\text { Coeficiente } \\
\text { angular } \\
( \pm \text { desvio } \\
\text { padrão })\end{array}$ & $\begin{array}{c}\mathrm{CL}_{50}\left(\mathrm{ppm}^{\mathrm{b}}\right) \\
(\mathrm{I} . \mathrm{C} .95 \%)\end{array}$ & $\begin{array}{c}\mathrm{CL}_{95}\left(\mathrm{ppm}^{\mathrm{b}}\right) \\
(\mathrm{I} . \mathrm{C} .95 \%)\end{array}$ & $\mathrm{X}^{2}$ & g.l. \\
\hline Lufenurom & 150 & $3,926 \pm 0,685$ & 0,265 & 0,697 & 3,681 & 8 \\
\hline Novalurom & 165 & $2,012 \pm 0,413$ & 0,066 & 0,437 & 11,151 & 9 \\
\hline Metoxifenozida & 120 & $3,311 \pm 0,964$ & $(0,049-0,084)$ & $(0,256-1,447)$ & & \\
\hline
\end{tabular}

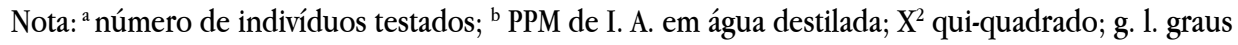
de liberdade.

Fonte: Dados da pesquisa 
A curva de concentração de mortalidade para lufenurom foi a que apresentou maior inclinação, de 3,926 $\pm 0,685$, seguido por metoxifenozida de 3,311 $\pm 0,964$, novalurom de 2,012 $\pm 0,413$, e espinosade de 1,971 $\pm 0,589$, que apresentou a menor inclinação (Tabela 2); valores altos de inclinação da curva indicam que pequenas variações na concentração do inseticida promovem grandes variações na mortalidade e vice-versa.

As informações obtidas nesse trabalho podem contribuir para o conhecimento do potencial tóxico dos produtos avaliados em $S$. frugiperda.

Conforme as Figuras 1 a 4, constata-se que a ação tóxica dos inseticidas pode ser representada da seguinte forma: novalurom $>$ lufenurom $>$ espinosade $>$ metoxifenozida. Desta maneira o inseticida com maior toxicidade, expressa por uma menor CL50, foi novalurom e de menor toxicidade foi metoxifenozida. Os inibidores da síntese de quitina (lufenurom e novalurom) apresentaram diferenças no valor de CL50. Lufenurom foi 4 vezes mais tóxico para lagartas de $S$. frugiperda do que o novalurom. Este resultado sugere que apesar do inseticida apresentar o mesmo modo de ação nas lagartas em estudo, são distintos sob o ponto de vista toxicológico. Estas diferenças são expressas principalmente através da formulação e concentração dos produtos.

O inseticida espinosade possui ação neurotóxica e pertence ao grupo das espinosinas, atuando como agonista da acetilcolina, nos receptores da célula pós-sinapse. Analisando-se a performance deste sobre os inseticidas do grupo dos reguladores de crescimento de insetos citados anteriormente, nota-se que espinosade possuiu uma toxicidade 14,7 vezes inferior em relação ao novalurom e até 3,6 vezes em relação ao lufenurom, considerando a CL50.

O metoxifenozida é um inseticida regulador de crescimento de insetos, porém apresenta um mecanismo de ação diferenciado quando comparado ao lufenurom e novalurom. Este mecanismo baseia-se na aceleração do processo de ecdise atuando como agonista de hormônios ecdisteróides (DHADIALLA et al., 1998). O metoxifenozida foi 68,8 vezes mais tóxico do que o novalurom, 17,1 vezes em relação ao lufenurom e 4,68 vezes que o espinosade.

Nota-se que dos produtos estudados, o metoxifenozida foi o menos tóxico, uma vez que necessitou de uma concentração 68,8 vezes maior do que o novalurom para causar uma mortalidade de $50 \%$ das larvas. 
Dentro do manejo integrado de pragas deve-se observar no controle de lagartas de $S$. frugiperda o estágio do milho. Desta maneira podem-se empregar inseticidas de contato em estágios iniciais e ingestão em estágios superiores a quatro folhas. Uma maior quantidade de folhas pode propiciar um desempenho melhor dos produtos devido à relação área foliar/eficiência da aplicação. Assim uma maior quantidade de folhas coberta com o inseticida empregado propicia um maior sucesso no controle pela ação de ingestão do inseticida pela praga.

Os inseticidas estudados no presente trabalho, todos apresentam DL50 oral maior do que $5.000 \mathrm{mg} / \mathrm{kg}$ (peso vivo). Então pode-se evidenciar que do ponto de vista toxicológico, são inseticidas ambientalmente mais seguros.

Cabe ressaltar que informações sobre a linha básica de suscetibilidade de $S$. frugiperda representam o primeiro passo de um programa preventivo de manejo da resistência (SCHMIDT, 2002). Os programas de monitoramento da suscetibilidade a inseticidas normalmente se baseiam em comparações entre doses letais e coeficientes angulares de curvas de dose-mortalidade estimadas para diferentes populações de um inseto-praga (TWNE; REYNOLDS, 1980).

Os resultados obtidos nesse trabalho servirão de base para estudos de doses subletais em lagartas de $S$. frugiperda, e também para estudos futuros sobre variações na sensibilidade da praga aos inseticidas testados.

\section{CONCLUSÃO}

O inseticida lufenurom apresentou CL50 $=0,265$ ppm (I. C. 95\% 0,215 0,309 ) e CL95 = 0,697 ppm (I. C. $95 \%$ 0,553 - 1,071), novalurom CL50 = 0,066 ppm (I. C. 95\% 0,049 - 0,084) e CL95 = 0,437 ppm (I. C. 95\% 0,256 - 1,447), metoxifenozida CL50 = 4,544 ppm (I. C. 95\% 3,569 - 5,574) e CL95 = 12,628 ppm (I. C. 95\% 8,681 - 41,184) e espinosade CL50 = 0,970 ppm (I. C. 95\% 0,638 - 1,382) e CL95 = 6,63 ppm (I. C. 95\% 3,195 - 96,545). Novalurom apresenta efeito tóxico a $S$. frugiperda maior que o lufenurom, que por sua vez é maior que espinosade e metoxifenozida. 


\section{AGRADECIMENTOS}

À Coordenação de Aperfeiçoamento de Pessoal de Nível Superior pela bolsa concedida aos autores.

\section{REFERÊNCIAS}

BARRERO, M. R. Prospecção e caracterização de genes de Bacillus Thuringiensis com potencial para o controle de insetos-praga da cultura da soja. 2005. $93 \mathrm{f}$. Tese (Doutorado em Ciências Biológicas) - Universidade Federal do Paraná, Curitiba, PR.

CARVALHO, R. P. L. Danos, flutuação da população, controle e comportamento de Spodoptera frugiperda (J.E. Smith, 1797) e suscetibilidade de diferentes genótipos de milho, em condições de campo. 1970. 170f. Tese (Doutorado). Escola Superior de Agricultura "Luiz de Queiroz", Universidade de São Paulo, Piracicaba.

CARVALHO, A. O. R. de. Pragas e seu controle. In: IAPAR. O milho no Paraná. Londrina: IAPAR, 1982. (Circular Técnica, 29).

CONAB. Acompanhamento de safra brasileira: grãos, quarto levantamento, janeiro 2014. Brasília, 2013. 28 p. Disponível em: < http://www.conab.gov.br/ OlalaCMS/uploads/arquivos/14_07_09_09_36_57_10_levantamento_de_graos julho_2014.pdf > Acesso em: 11 jul. 2014.

CRUZ, I.; VIANA, P. A.; WAQUIL, J. M. 1999. Manejo das pragas iniciais de milho mediante o tratamento de sementes com inseticidas sistêmicos. Sete Lagoas: EMBRAPA-CNPMS, 1999. 39f. (EMBRAPA-CNPMS. Circular Técnica, 31).

CRUZ, I. Manejo de pragas da cultura do milho. In: CRUZ, J. C.; KARAM, D.; MONTEIRO, M. A. R.; MAGAlHAES, P. C. (Ed.). A Cultura do Milho. Sete Lagoas: Embrapa Milho e Sorgo. Cap. 12, p. 303-362, 2008. 
DHADIALLA, T. S.; CARLSON, G. R.; LE, D. P. New insecticides with ecdysteroidal and juvenile hormone activity. Annual Review of Entomology, v. 43, p. 545-569, 1998.

FINNEY, D. J. Probit analysis. Cambridge: Cambridge University Press, 1971. 255p.

GASSEN, D. N. Pragas associadas à cultura do milho. Passo Fundo: Aldeia Norte. 92p. 1994.

GREENE, G. L.; LEPPLA, N. C.; DICKERSON, W. A. Velvetbean caterpillar: a rearing producedure and artificial medium. Journal Economic Entomolog y, v. 69, n. 4, p. 487-497, 1976.

OMOTO, C. Modo de ação de inseticidas e resistência de insetos a inseticidas. In: GUEDES, J. C.; COSTA, I. D.; CASTIGLIONI, E. Bases e técnicas do manejo de insetos. Santa Maria: UFSM/CCR/DFS; Palloti, 2000, p. 31-49.

SILVA, A. G. d'A; GONÇALVES, C. R.; GALVÃO, D. M. et al. Quarto catálogo dos insetos que vivem nas plantas do Brasil, seus parasitos e predadores. Rio de Janeiro: Ministério da Agricultura, 1968. v. 1, pt. 2, 622p.

SCHMIDT, F. B. Linha básica de suscetibilidade de Spodoptera frugiperda (Lepidoptera: Noctuidae) a lufenuron na cultura do milho. 2002. 48f. Dissertação (Mestrado em Entomologia) - Escola Superior de Agricultura Luiz de Queiroz, Piracicaba, 2002.

TWINE, P. H.; REYNOLDS, H. T. Relative susceptibility and resistance of the tobacco budwoem to methyl parathion and synthetic pyretrhoids in southern California. Journal of Economic Entomology, v. 73, p. 239-242, 1980.

Recebido em: 08 de julbo de 2015 Aceito em: 22 de dezembro de 2015 